Supporting Information from

\title{
Colloidal stability and concentration effects on nanoparticle heat delivery for magnetic fluid hyperthermia
}

\author{
Vanessa Pilati, ,,‡ Guilherme Gomide, ${ }^{\ddagger}$ Rafael Cabreira Gomes, $₫$ Gerardo \\ F.Goya, ${ }^{\S}$ and Jérôme Depeyrot ${ }^{\ddagger}$ \\ ‡Complex Fluids Group, Instituto de Física, Universidade de Brasília, Caixa Postal \\ 04455,70919-970, Brasília, DF, Brazil \\ "Departamento de Física, Universidade Federal de Santa Catarina, 88040-900, \\ Florianópolis, SC, Brazil. \\ §Instituto de Nanociencia y Materiales de Aragón (INMA), Universidad de Zaragoza, \\ 50018, Zaragoza, Spain.
}

Number of pages: 3

Number of figures: 2

Number of tables: 2 


\section{Crystalline structure}

The crystalline structure of the NPs before (bare NPs) and after the surface treatment with iron nitrate (core/shell NPs) was investigated by XRPD measurements. The obtained diffractogram patterns are depicted in Figure SI-1 and the observed peaks are correspondent to the cubic spinel structure. Crystalline diameters are obtained by using Scherrer's formula applied to [311] peak, which is the most intense peak one. The lattice parameters are obtained by using the mean value obtained by the 6 most intense peak from the diffractograms. Results are depicted in Table 1 of the main manuscript.
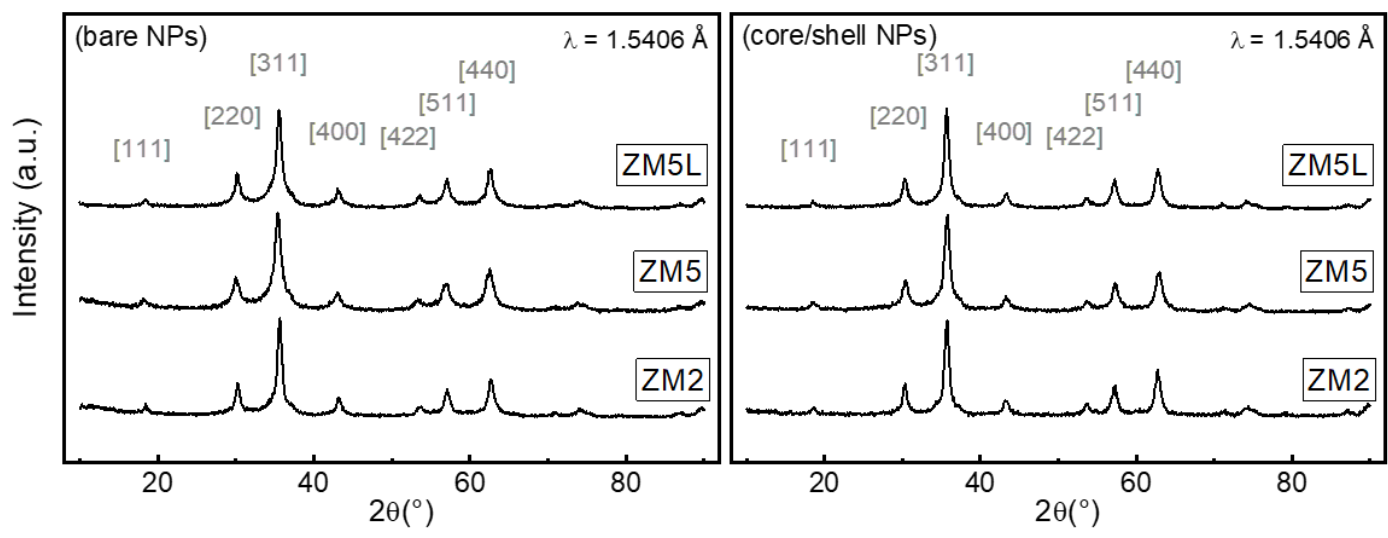

Figure SI-1 - XRP Diffractograms of the studied samples before (left) and after (right) the surface treatment with iron nitrate.

\section{SAXS Calculations}

Table SI-1 - Size distribution parameters utilized in the SAXS form factor determination and a comparative with TEM results.

\begin{tabular}{ccccc}
\hline Sample Name & $\mathrm{d}_{0}$ SAXS & $\mathrm{s}^{\text {SAXS }}$ & $\mathrm{d}_{0}{ }^{\text {TEM }}$ & $\mathrm{s}^{\text {TEM }}$ \\
\hline ZM2 & 10.2 & 0.39 & 9.2 & 0.30 \\
ZM5L & 7.2 & 0.50 & 7.2 & 0.44 \\
ZM5 & 7.8 & 0.43 & 7.4 & 0.35 \\
\hline
\end{tabular}




\section{Magnetic hyperthermia}

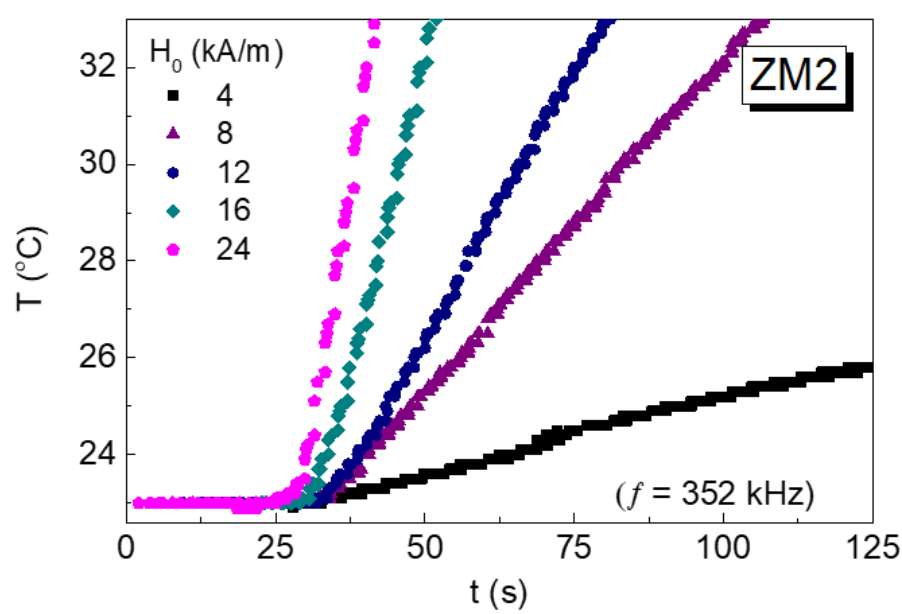

Figure SI- 2- Representative heating curves for measurements with frequency of $352 \mathrm{kHz}$ and different magnetic field amplitudes. Measurements for 10 $\mathrm{mg} / \mathrm{mL}$ of ZM2 NPs dispersed with $\mathrm{pH}=2.0$.

Table SI-2 - Results from LRT fitting in SPA $x$ frequency experiments for NPs dispersed in water $(\mathrm{pH}=2.0)$ and agarose gel $(\mathrm{pH}=7.0)$. Both with concentrations with $\sim 49 \mathrm{mg} / \mathrm{mL}$.

\begin{tabular}{ccccc}
\hline Sample & medium & $\mathrm{a}(\mathrm{J} / \mathrm{g})$ & $\begin{array}{c}\tau(\mathrm{s}) \\
\left(10^{-7} \mathrm{~s}\right)\end{array}$ & $\begin{array}{c}\mathrm{K}_{\text {eff }}{ }^{*} \\
\left(\mathrm{~J} / \mathrm{m}^{3}\right)\end{array}$ \\
\hline ZM2 & water $\mathrm{pH}=2.0$ & $1.4 \times 10^{-4}$ & 3.2 & $8.9 \times 10^{3}$ \\
ZM2 & agarose $\mathrm{pH}=7.0$ & $1.0 \times 10^{-4}$ & 4.2 & $9.3 \times 10^{3}$ \\
\hline ZM5L & water $\mathrm{pH}=2.0$ & $4.2 \times 10^{-5}$ & 2.0 & $1.2 \times 10^{4}$ \\
ZM5L & agarose $\mathrm{pH}=7.0$ & $3.8 \times 10^{-5}$ & 2.1 & $1.2 \times 10^{4}$ \\
\hline
\end{tabular}

